\title{
Integralidade do cuidado de enfermagem do pré-natal ao puerpério
}

\section{Completeness of nursing care from prenatal to puerperium}

Beatriz Assunção Ferreira ${ }^{1}\left({ }^{0}\right.$, Elizabeth Mesquita da Silva ${ }^{1}\left(\mathbb{0}\right.$, Adriano da Costa Belarmino² ${ }^{\mathbb{C}}$, Rosana Gomes de Freitas Menezes Franco ${ }^{3}$ (D) , Isabelle Cordeiro de Nojosa Sombra ${ }^{4}$ (D) , Alisson Salatiek Ferreira de Freitas ${ }^{3,5}$ (D)

1. Discente do Curso de Enfermagem, Centro Universitário Christus (UNICHRISTUS), Fortaleza, CE, Brasil. 2. Mestre em Saúde Coletiva, Universidade Estadual do Ceará (UECE), Fortaleza, CE, Brasil. 3. Docente Curso de Enfermagem, Centro Universitário Christus (UNICHRISTUS), Fortaleza, CE, Brasil. 4. Doutoranda em Obstetrícia pela Universidade Federal de São Paulo (UNIFESP), São Paulo, SP, Brasil. 5. Doutorando em Saúde Coletiva pela Universidade Estadual do Ceará (UECE), Fortaleza, CE, Brasil.

\section{Resumo}

Objetivo: compreender a integralidade do cuidado de enfermagem do pré-natal ao puerpério. Método: pesquisa de campo, com abordagem qualitativa, realizada na unidade hospitalar, localizada na região metropolitana de Fortaleza/CE. A coleta de dados ocorreu no período de abril a maio de 2021 , com a participação de 26 puérperas. Resultados: a mulher, no tocante à sua integralidade durante o ciclo do pré-natal ao puerpério, obteve um cuidado com o foco biológico, surgindo a família como um apoio para os aspectos emocionais. 0 enfermeiro é o profissional responsável pela criação da confiabilidade da gestante e família, durante o momento do pré-natal até o puerpério. Conclusão: a integralidade do cuidado desenvolvido pelo enfermeiro encontra desafios relacionados à necessidade de uma visão que contemple o processo saúde-doença que rodeia essa mulher.

Palavras-chave: Saúde da Mulher; Gravidez; Cuidados de Enfermagem; Período Pós-parto.

\begin{abstract}
Objective: To understand the comprehensiveness of nursing care from prenatal care to puerperium. Method: Field research, with a qualitative approach, carried out in a hospital located in the metropolitan region of Fortaleza/CE. Data collection took place from April to May 2021 with the participation of 26 postpartum women. Results: The woman, regarding her completeness during the cycle from prenatal to postpartum obtained a care with the biological focus, emerging the family as a support for the emotional aspects. The nurse is the professional responsible for creating the reliability of the pregnant woman and her family, from the prenatal period to postpartum. Conclusion: The integrality of care provided by nurses faces challenges related to the need for a vision that contemplates the health-disease process that surrounds this woman.
\end{abstract}

Keywords: : Women's Health; Pregnancy;Nursing Care; Postpartum Period

\section{INTRODUÇÃO}

O período gestacional é a formação de um novo ser, iniciado na fecundação, o qual atinge cerca de 41 semanas e termina com o parto; é marcado por alterações no estilo de vida da mulher, provocando mudanças na vida pessoal, do casal e da família. $\mathrm{Na}$ assistência do período pré-natal, é fundamental ofertar uma experiência positiva na gestação e no puerpério, por meio da promoção do cuidado materno respeitoso, individualizado e centrado na mulher a cada contato, com a implementação de práticas efetivas e informações relevantes em momento oportuno, assim como por meio do suporte emocional e psicossocial $^{1}$.

Assim, os cuidados prestados à mulher no processo de parturição sofreram modificações significativas ao longo dos anos, sendo necessária a criação de políticas públicas para implantar a humanização ao parto, em prol de reduzir o número de cesárias e regulamentar o acompanhamento do trabalho de parto, oferecendo descobertas no campo da ciência e tecnologia para controlar complicações e possíveis condições de risco materno e fetal ${ }^{2}$.
Sabe-se que a gestação para mulher e sua família é a fase mais importante na vida, na qual ocorrem várias mudanças físicas e, principalmente, emocionais, sendo considerada uma fase de vulnerabilidade dessa gestante. É nesse período que, no pré-natal, construímos um vínculo entre o profissional e a mãe, adaptando o real papel materno e transformando esse momento estressante em compensatório para ela, seu companheiro, sua família e cuidador ${ }^{3}$.

As ações de saúde na atenção à mulher e à criança são prioridades nas políticas públicas para a redução dessa mortalidade. No século XX e nos anos 2000, o Brasil diminuiu a mortalidade infantil alcançando, em 2018, 14,4 óbitos a cada 100 mil crianças, menores de 5 anos nascidas vivas. Os óbitos maternos apresentaram redução, mas 60 óbitos a cada 100 mil nascidos vivos em 2015 foram insuficientes para o Brasil atingir a meta de reduzir o valor a 35 óbitos a cada 100 mil nascidos vivos, conforme previsto nos Objetivos de Desenvolvimento do Milênio4. 
É nesse contexto que a integralidade do cuidado de gestantes desde o pré-natal, parto e pós-parto abre um leque de reflexões ao relatar que o princípio do Sistema Único de Saúde - SUS se faz por completo nas práticas de saúde, que visam à condição integral, e não parcial, de compreensão do ser humano, ou seja, inserem o usuário em seu contexto social e, a partir daí, atendem às demandas e necessidades dessa pessoa ${ }^{5}$.

Nesse contexto, o Ministério da Saúde (MS) criou a Portaria de no 569/2000 que visa a melhorias para a assistência ao parto humanizado nos serviços de saúde, assegurando o acesso, a cobertura e a qualidade do acompanhamento dessas gestantes. O pré-natal, a assistência ao parto e puerpério e a assistência neonatal juntamente com o Programa de Humanização no Pré-natal e Nascimento (PHPN) são realizados na Atenção Primária, no programa Estratégia de Saúde da Família (ESF), por enfermeiros respaldados pela Resolução do Conselho Federal de Enfermagem/Cofen, no 627/20206.

O enfermeiro é o profissional fundamental para efetuar um pré-natal qualificado, pois traça estratégias de promoção à saúde, prevenção e humanização juntamente com a gestante e a família ${ }^{7}$, cuidando das necessidades identificadas e proporcionando à gestante a resolução desse parto, orientando-a aos cuidados com o recém-nascido, orientando e encaminhando para serviços de saúde da criança para cuidados no puerpério saudável e eficaz.

O interesse do estudo surgiu da vivência de estágio extracurricular, ao observar a equipe de enfermagem proporcionar à parturiente um desenvolvimento saudável da gestação e do momento do parto, fornecendo informações e alívio à dor, promovendo saúde materno infantil utilizadas durante o pré-natal até o puerpério, influenciando na iniciação de um puerpério seguro e envolvendo conhecimentos estruturados quanto ao desenvolvimento da prática da enfermagem no centro obstétrico.

Baseado neste contexto, surgiu o seguinte questionamento: como se processa a integralidade do cuidado da mulher pela enfermagem no pré-natal até o momento do puerpério? Pressupõe-se que a enfermagem age durante esse período gravídico trabalhando ações no contexto biológico, social, psicológico e físico, capacitando-as durante o trabalho de parto e transformando-as como as grandes protagonistas deste momento.

Assim, o presente estudo desenvolveu-se com o objetivo de compreender a integralidade do cuidado de enfermagem do pré-natal ao puerpério, descrevendo a visão das puérperas sobre a integralidade dos cuidados de enfermagem prestados no pré-natal, parto e puerpério e identificando as ações de cuidados do enfermeiro prestados durante o pré-natal, parto e puerpério.

\section{METODOLOGIA}

Este estudo tratou de uma pesquisa de campo, descritiva, transversal com abordagem qualitativa que foi realizada no Hospital Municipal Dr. Amadeu Sá, localizada na Rua Carmelita Rebouças, 266 em Eusébio, região metropolitana da cidade de Fortaleza/ CE, na região Nordeste do Brasil. É uma unidade hospitalar de nível secundário referenciado em acolher gestantes não só da cidade, mas de toda a região próxima a ela. A unidade hospitalar é composta por 28 leitos, separada por 5 enfermarias obstétricas, sendo 11 leitos de parto humanizado e 17 leitos para partos cesáreos, onde é oferecido o acompanhamento às gestantes e puérperas, o qual com uma equipe multiprofissional para um acompanhamento no trabalho de parto e parto de modo humanizado, baseado em evidências em gestações sem intercorrências.

As participantes do estudo foram puérperas que realizaram seu parto na cidade de Eusébio-CE, acompanhadas no pré-natal e encaminhadas para o momento de seu parto. Elas foram incluídas por critérios de admissão na unidade hospitalar em seu terceiro trimestre gestacional, tendo realizado, no mínimo, três consultas com o enfermeiro no pré-natal. Foram excluídas gestantes menores de 18 anos de idade e/ou com estratificação de alto risco.

Foram entrevistadas 26 puérperas após as suas 24 horas pósparto, sendo o quantitativo de participantes definidos pela recorrência ou saturação dos dados adquiridos por meio das falas dos sujeitos entrevistados, ou seja, quando as informações não mais apresentarem novo conteúdo ${ }^{8}$.

A coleta de dados ocorreu no período de abril a maio de 2021, por meio de um roteiro de entrevistas semiestruturadas, que teve uma duração de 3 a 9 minutos, sendo elas gravadas e transcritas, compostas por questões socioeconômicas, dados clínico e questionamentos sobre seus sentimentos e como percebem a assistência de enfermagem no pré-natal, parto e puerpério.

Os dados foram analisados por meio de sua caracterização8, cujos resultados foram transcritos no Microsoft Word; os dados coletados foram processados, verificados e avaliados. A partir da saturação das falas, surgiram as categorias temáticas: ressignificando a gestação, o parto e o puerpério, e o ser enfermeiro e o processo de cuidado da gestação, parto e puerpério.

0 estudo foi totalmente realizado em cumprimento às normas da Resolução no 466/12 do Conselho Nacional de Saúde, responsável pelos princípios éticos e científicos fundamentais de pesquisas com seres humanos (9), sendo aprovado pelo Comitê de Ética do Centro Universitário Christus, com o Parecer de $\mathrm{n}$ - 4.605.241.

\section{RESULTADOS E DISCUSSÕES}

Conforme as entrevistas, a variação de idade é de 13 com faixa etária de 21 a 29 anos de idade, e 13 são de faixa etária entre 30 a 39 anos de idade. A situação conjugal foi descrita como 7 casadas, 11 mães solteiras, 7 em união estável e 1 divorciada. A 
escolaridade entre elas variou da seguinte forma: 3 terminaram o ensino fundamental completo, 18 concluíram o ensino médio e 5 finalizaram o ensino superior; 3 relataram que recebem menos de um salário-mínimo; 15 relataram uma renda familiar de um a dois salários-mínimos, 5 com renda familiar de mais de dois salários-mínimos.

Conforme a idade gestacional na admissão no hospital, 19 gestantes foram admitidas na unidade entre 37 a 39 semanas de gestação, e só sete delas entraram com 40 a 41 semanas de gestação. Destas, 25 foram resoluções de via de parto cesáreo, sendo duas desses partos gestações gemelares, uma por via de parto normal, e 24 não apresentaram intercorrências durante o parto.

Aos históricos obstétricos, 11 eram primíparas e 15 multíparas. Todas informaram que a realização do seu pré-natal ocorreu no serviço público, sendo que 26 realizaram de 4 a 19 consultas de pré-natal em que 23 puérperas realizaram de 3 a 8 consultas com enfermeiro da unidade básica de saúde, e só 3 realizaram de 0 a 2 consultas com enfermeiro no seu pré-natal; 19 se autodeclararam pardas, uma considerou-se branca, duas admitem-se negras, e apenas uma se declarou como indígena; 11 disseram ser católicas, 10 evangélicas e cinco não têm uma religião; 10 residem em Eusébio, seis em Fortaleza, quatro em Itaitinga; uma em Cascavel, uma em Beberibe, uma em Chorozinho; uma em Barreira e duas em Paraipaba.

É importante mencionar que, no pré-natal, existem estratégias sendo implementadas para minimizar os índices de morte materna e fetal, como o diagnóstico precoce de possíveis patologias e seu tratamento oportuno, que asseguram uma gestação segura, um bom desenvolvimento fetal, chegando a evitar em $98 \% 98 \%$ das mortes maternas ${ }^{10}$.

\section{RESSIGNIFICANDO A GESTAÇÃO, PARTO E PUERPÉRIO}

O pré-natal é caracterizado por ações que identificam, de forma adequada e precoce, pacientes com evolução desfavorável e acolhem-nas desde o princípio da gestação, escutandoas atentamente e transmitindo-Ihes confiança para que conduzam a gestação com autonomia até o parto ${ }^{11}$. É um período caracterizado por mudanças físicas e emocionais que, consequentemente, devem-se agregar às estratégias que garantam que a gestante seja assistida de forma integral pelas equipes de saúde.

Assim, podemos observar que E4 e E12 apontaram necessidades de acompanhamento profissional não somente no aspecto biológico, mas contemplando o emocional e social, como podemos ver nas seguintes falas:

E4: “Eu achei que não seria capaz, porque meu corpo não consegue segurar para ter um parto normal, eu quis na primeira vez, mas não teve preparação. " e E12: "O meu não foi muito tranquilo, porque aconteceram algumas questões familiares que acabaram atrapalhando, meu esposo sofreu uma tentativa de homicídio."

Durante o acompanhamento pré-natal, as ações dos profissionais tendenciam a abordar muito mais os problemas físicos em detrimento do bem-estar psicológico e emocional das mães, mas a dimensão emocional e psíquica da gestante requer atenção pela percepção exclusiva de alguma alteração mental, como sintomas de depressão ou transtorno de ansiedade ${ }^{12}$. Afinal, este processo gestacional não acaba nas últimas consultas de pré-natal.

Durante as coletas, foi investigado se, por algum momento, foi trabalhada a situação de apoio emocional das entrevistadas, uma vez que é um dos fatores de mudança na gestação. Obtendo as seguintes falas:

E14: "Só entre eu e meu marido." e pela E20: “Não, mas eu precisei principalmente nessa pandemia, sempre fui muito de assistir jornal desde criança, e tive muitos choros de ansiedade, essa gravidez foi muito difícil, então tinha dias que era muito difícil."

As falas evidenciam uma fragilidade no acompanhamento emocional das gestantes, por meio da equipe que acompanha seu pré-natal até o momento do parto, pois elas sofrem interferências sociais e ambientais, muitas vezes encontrando como apoio somente seu parceiro. Associado ao problema cotidiano da mulher, durante esse período, outros fatores estressores, motivados pela pandemia do COVID-19, estiveram interferindo a saúde materna-infantil, trazendo incertezas sobre o que irá ocorrer no futuro.

Planejar o parto favorece a preparação da mulher durante o trabalho de parto, pois aprimora a efetividade e o empoderamento com a presença do acompanhante durante o pré-natal, parto e puerpério, contribuindo nos desfechos maternos e neonatais. Então, todos os esforços devem ser realizados para garantir que, neste momento, haja uma pessoa de sua escolha para encorajá-la e dar-lhe conforto durante todo o processo ${ }^{13}$.

A relação profissional-usuária, que se estabelece durante a assistência, baseada no diálogo, nas informações e orientações sobre os cuidados em saúde, é um diferencial que contribui para o alcance da qualidade ${ }^{14}$; desse modo, é necessária a permanência dessa assistência para a redução das taxas de morbimortalidade materna e perinatal.

O Programa de Humanização no Pré-natal e Nascimento pela Rede Cegonha assegura a integralidade dos cuidados com promoção e prevenção no período gestacional e pósparto, preparando as gestantes para um puerpério saudável e tranquilo.

Em relação ao atendimento desenvolvido no pré-natal, podese observar que o sistema estratifica as gestantes buscando desenvolver um acompanhamento mais intenso e contínuo 
segundo sua necessidade, como afirma E6:

"Fui pro posto, e como era de risco, eles me encaminharam pra policlínica do Hospital da Mulher, [...], eu ia de 15 em 15 dias, (...) tive muita orientação, tinha psicóloga, tinha nutricionista, tinha endócrino, a preocupação deles era bem. "

Além da estratificação, a mulher, em seu pré-natal, encontra apoio para se preparar para as atividades que a maternidade traz para família, bem como a garantia da realização de exames como relata a $\mathrm{E} 18$ :

"Lá eu tive o sistema de amamentação, como que eu amamento de forma certa, as enfermeiras me explicavam tudo, os exames que eu ia fazer, e eu fiz todos pelo SUS.".

Apesar desse acompanhamento e suporte oferecido gratuitamente para as gestantes, três participantes apontaram a mesma situação relatada pela E16:

(...) Não, na medida do possível que o SUS poderia me oferecer e fornecer foi suprido, entendeu [...]. A solicitação de exames, como os testes rápidos, eles tinham gratuitos né, alguns né, nem todos, porque tem a questão da agilidade (...).

Apesar da ampliação no acompanhamento na assistência do prénatal, há incidentes de morbimortalidade materna e perinatal, ocasionadas por complicações advindas da hipertensão arterial e/ou da diabetes gestacional, necessitando de tomadas de decisão e condutas terapêuticas que, muitas vezes, o tempo de resposta para investigação de exames não responde com a rapidez desejada, reduzindo que essas complicações sejam tratadas e acompanhadas ainda durante o pré-natal ${ }^{15}$.

As Síndromes Hipertensivas na Gravidez (SHG) comprometem o período gravídico e precisam ser prevenidas, assegurando a essas gestantes cuidados especializados, durante e após o parto, devendo-se postergar-se em todo o período gestacional, abrangendo todas as etapas e equipe multiprofissional. Muitas dessas síndromes podem afetar a dinâmica normal da mulher como relata a E6:

"Foi diferente da primeira vez, (...) desde os 4 meses que eu to afastada. [...] por conta da minha pressão, tive começo de eclâmpsia, tive muitas infecções urinárias, essas coisas né."

A integralidade do cuidado de enfermagem na gestação caracteriza-se como a integração de ações, inclui a promoção da saúde e prevenção de doenças (Diabetes Gestacional e Hipertensão Gestacional) que afetam gestantes com histórico familiar e maus hábitos de vida. A integralidade pressupõe a articulação da saúde com outras políticas públicas, garantindo atuações intersetoriais entre as diferentes áreas na saúde e qualidade de vida para as gestantes ${ }^{16}$.

Assim, durante as coletas sobre a assistência pré-natal, observou-se que boa parte das gestantes se usufruía de informações, conhecimentos e orientações sobre possíveis complicações que percorreram a saúde da mulher durante esse período, complicações estas que podem resultar na saúde do binômio, formando vínculos que percorreram até o pós-parto incluindo a saúde da criança, fortalecendo essa relação entre mãe, filho e profissional.

\section{O SER ENFERMEIRO E O PROCESSO DE CUIDADO DIANTE DA GESTAÇÃO, PARTO E PUERPÉRIO}

As consultas de enfermagem no pré-natal de baixo risco é o momento para a troca de informações e vínculo entre o profissional responsável e a gestante, com o objetivo que ela tenha um desenvolvimento gestacional saudável (17). Desse modo, almejando um vínculo entre o enfermeiro e a usuária, ficam bem evidenciados nas falas da E2

"Tive bastante orientação, no que deveria comer e o que não deveria né, e sobre as vitaminas que eu precisava tomar na minha gestação". e E21: “(...) As consultas que eu tinha eram boas, porque eu ia tirando algumas dúvidas com a enfermeira, foi mais uma troca de conhecimento entre eu e ela (...).",

Essas condutas e os cuidados de enfermagem no pré-natal durante $\mathrm{O}$ acompanhamento dessa gestação, visa diminuir as complicações materno fetal.

Na Rede Cegonha, a gravidez e pós-parto preconizam ações de prevenção e promoção à saúde, além de diagnóstico e tratamento adequado dos problemas que ocorrem neste período (18). Diante desse cenário, as gestantes têm receio de agravos neste período da gestação e até o parto, como a possibilidade de transmissão do vírus, afetando os serviços de saúde(19). Com objetivos de permitir a autonomia da gestante no momento do parto e puerpérios, a educação em saúde tornase um forte aliado do enfermeiro, promovendo tranquilidade, confiança e vínculo na relação enfermeiro-mulher, como relata a E24:

[...] ela explica tudo, o que tá acontecendo e o que não tá, eu tirei muitas dúvidas com ela. e na fala da E25: Foi ótima minhas consultas[...] Ela sempre me explicou as coisas, e ela também já tinha acompanhado minha primeira gestação, então foi ótimo.

O cuidado prestado durante a gestação, o parto e puerpério, a interação e a comunicação entre o profissional e a gestante tomam conta do momento, com o planejamento na assistência em saúde da mulher e sua família (20), traçando ações que ativam a rede de apoio na tomada de decisão, no momento do nascimento, podendo trazer a ressignificação das ações do ser 
enfermeiro mediante o momento do parto para a mulher e a família.

Torna-se visível, durante as coletas das informações, presente em um dos fatores para uma assistência de qualidade e formação de vínculo vantajoso entre profissional e gestante, a comunicação clara e direta no momento mais esperado da gestação dessa mulher, que é o parto, como afirma E21:

"Se fosse pra dar uma nota, eu daria 10, porque foi muito tranquilo e fui bem acolhida sempre, em nenhum momento eu me senti só, e no posto também eu sempre me senti bastante acolhida, não deixaram nada a desejar!"

O momento do parto é um evento de caráter íntimo que, com o passar dos anos, ocorrem mudanças que se transformam com o tempo, trazendo a valorização da mulher como protagonista do parto. Deve-se ressaltar que humanizar o parto não é só ter um parto normal, realizando ou não procedimentos, mas também tornar a mulher protagonista daquele momento e não apenas espectadora ${ }^{21}$.

O puerpério gera repercussões na gravidez e no parto, podendo estar presentes nas mulheres até um ano pós-parto com transformações físicas e psíquicas na mulher, tornando-as susceptíveis ao surgimento de agravos neste período ${ }^{22}$. Esta puérpera deve ser referenciada para a unidade do pré-natal, com relatório completo sobre o nascimento e o pós-parto imediato.

Ainda no ambiente hospitalar, começa o grande desafio dessa puérpera, em meio as outras puérperas e seus neonatos como relata nove das entrevistadas, sendo representada pela fala da E3:

\footnotetext{
"Mais ou menos, porque aqui minha acompanhante teve que ir embora, e eu to sozinha agora né, mas acho que lá em casa eu vou ter mais ajuda pra cuidar dele.".
}

Com as alterações biológicas e a deficiência no padrão de sono, necessitará de bastante apoio do acompanhante e que essa assistência com o neonato percorrerá para unidade de saúde onde acompanhou seu pré-natal.
Logo, a vulnerabilidade em que essas mulheres se encontram diante da nova mudança de rotina e os desafios para o cuidado com o recém-nascido geram uma tendência para o déficit do autocuidado, que, consequentemente, poderá influenciar no cuidado com o neonato. Assim, a equipe do serviço de saúde na atenção primária deve ficar sob alerta para o acolhimento do binômio, assegurando a integralidade do cuidado para com eles.

\section{CONSIDERAÇÕES FINAIS}

Mediante os resultados do estudo, conclui-se que o profissional enfermeiro se faz presente nos três momentos estudados: pré-natal, parto e puerpério, tendo atuação direcionada aos aspectos biológicos da mulher, fragilizando a atuação do cuidado integral ao alcance das necessidades reais da mulher diante das várias vertentes que envolvem o processo saúde-doença. Entretanto, foi constatado que o profissional enfermeiro desenvolve e fortalece seu vínculo com a mulher pelo processo de educação em saúde, contribuindo para a compreensão dos processos biológicos que envolvem a mulher na gestação, no parto e puerpério.

O estudo aponta, ainda, que a família é um importante instrumento de apoio, tanto relacionado ao contexto intrínseco como extrínseco da mulher no momento da gestação, do parto e puerpério, tornando-se significativo que o profissional enfermeiro contemple essa rede de apoio como processo de integralidade do cuidado.

A integralidade e a garantia do serviço de saúde para as mulheres no ciclo do pré-natal ao puerpério foram observadas como condizentes com as políticas de saúde da mulher; no entanto, foi observado que o tempo para espera dos resultados dos exames ainda é um entrave no acompanhamento do cuidado.

A pesquisa tem como fragilidade a coleta de dados ter ocorrido durante o período de pandemia, podendo os resultados ter sofrido influência pela dinâmica de saúde adotada. Entretanto, o estudo traz resultado significativo e reflexivo em relação à assistência integral e sugere o desenvolvimento de uma investigação mais ampla envolvendo os profissionais em busca de comparar os dados e buscar encontrar soluções para os entraves apontados neste estudo.

\section{REFERÊNCIAS}

1. Salvetti MG, Lauretti LG, Muniz RC, Dias TYSF, Oliveira AADG, Gouveia LMR. Characteristics of pregnant women at risk and relationship with type of delivery and complications. Rev Bras Enferm. 2021;74(4): e20200319. doi: https://doi. org/10.1590/0034-7167-2020-0319.

2. Guttier MC, Tejada CAO, Wehrmeister FC, Silveira MF, Domingues MR, Barros AJD et al. Gastos com a assistência ao parto: comparação entre as coortes de nascimento de Pelotas dos anos de 2004 e 2015. Cad Saúde Pública. 2020; 36(7): e00120019. doi: https://doi.org/10.1590/0102-311X00120019.

3. Bittencourt SDA, Cunha EM, Domingues RMSM, Dias BAS, Dias MAB, Torres
JA, Leal MC, et al. Nascer no Brasil: continuity of care during pregnancy and postpartum period for women and newborns. Rev Saúde Pública. 2020, 54: 100. doi: https://doi.org/10.11606/s1518-8787.2020054002021.

4. França EB, Lansky S, Rego MAS, Malta DC, França JS, Porto D et al. Principais causas da mortalidade na infância no Brasil, em 1990 e 2015: estimativas do estudo de Carga Global de Doença. Rev bras epidemiol. 2017 Maio, 20(Suppl 01): 46-60. doi: https://doi.org/10.1590/1980-5497201700050005.

5. Franco TB, Hubner LC. A Atenção Básica e os cuidados intermediários: um debate necessário. Saúde debate. 44(125): 516-526. doi: https://doi. 
org/10.1590/0103-1104202012518.

6. Ministério da Saúde [BR]. Portaria no 569, de 1o de junho de 2000. Institui o Programa de Humanização no Pré-natal e Nascimento, no âmbito do Sistema Único de Saúde [Internet]. Brasília, DF; 2000 [acesso 2021 Jul 5]. Disponível em: https://bvsms.saude.gov.br/bvs/saudelegis/gm/2000/prt0569_01_06_2000_ rep.html.

7. Gomes CBA, Dias RS, Silva WGB, Pacheco MAB, Sousa FGM, Loyola CMD. Prenatal nursing consultation: Narratives of pregnant women and nurses. Texto contexto - enferm. 2019; 28: e20170544. doi: https://doi.org/10.1590/1980265X-TCE-2017-0544.

8. Minayo MCS, Diniz D, Gomes R. O artigo qualitativo em foco. Ciênc saúde colet. 2016 Ago; 21(8): 2326. doi: https://doi.org/10.1590/141381232015218.15592016

9. Brasil. Resolução no 466, de 12 de dezembro de 2012. Aprova diretrizes e normas regulamentadoras de pesquisa envolvendo seres humanos [Internet]. Diário Oficial da União. 2012 Dez [acesso 2021 Jul 5]. Disponível em: https:// bvsms.saude.gov.br/bvs/ saudelegis/cns/2013/res0466_12_12_2012.html.

10. Leal NJ, Barreiro MSC, Mendes RB, Freitas, CKAC. Assistência ao pré-natal: depoimento de enfermeiras. Rev. Pesqui. 2018 Jan-Mar; 10(1): 113-122. doi: http://dx.doi.org/10.9789/2175-5361.2018.v10i1.113-122.

11. Andrade UV; Santos JB; Duarte C. A percepção da gestante sobre a qualidade do atendimento pré-natal em UBS, Campo Grande, MS. Rev. Psicol. Saúde. 2019; 11(1): 53-61. doi: http://dx.doi.org/10.20435/pssa.v0i0.585.

12. Costa DO, Souza FIS, Pedroso GC, Strufaldi MWL. Transtornos mentais na gravidez e condições do recém-nascido: estudo longitudinal com gestantes assistidas na atenção básica. Ciênc. saúde colet. 2018 Mar; 23(3): 691-700. doi https://doi.org/10.1590/1413-81232018233.27772015.

13. Holanda SM, Castro RCMB, Aquin PS, Pinheiro AKB, Lopes Martins, ES. Influência da participação do companheiro no pré-natal: Satisfação de primíparas quanto ao apoio no parto. Texto contexto - enferm. 2018; 27(2): e3800016. doi: https://doi.org/10.1590/0104-070720180003800016.

14. Livramento DVP, Backes MTS, Damiani PR, Castillo LDR, Backes DS, Simão
AMS. Percepções de gestantes acerca do cuidado pré-natal na atenção primária à saúde. Rev. Gaúcha Enferm. 2019, 40:e20180211. Disponível em doi: https:// doi.org/10.1590/1983-1447.2019.20180211.

15. Damasceno AAA, Malta MB, Neves PAR, Lourenço BH, Bessa ARS, Rocha DS et al. Níveis pressóricos e fatores associados em gestantes do Estudo MINABrasil. Ciênc saúde coletiva. 2020 Nov; 25(11): 4583-4592. doi: https://doi. org/10.1590/1413-812320202511.31742018.

16. Ministério da Saúde [BR]. Protocolos da Atenção Básica: Saúde das Mulheres. Brasília: Ministério da Saúde; 2016 [acesso 2021 Jul 5]. p. 73-230. Disponível em: https://bvsms.saude.gov.br/bvs/publicacoes/protocolos_atencao_basica_ saude mulheres.pdf.

17. Aparecida Maciel Cardelli A, Li Marrero, T; Ferrari RAP, Martins JT, Serafim D. Expectativas e satisfação das gestantes: desvelando o pré-natal na atenção básica. Investir. educ. enferm. 2016; 34(2): 252-260. doi: http://dx.doi. org/10.17533/udea.iee.v34n2a04.

18. Tomassi E, Fernandes PAA, Fischer T, Siqueira FCV, Silveira DS, Thumé E et al. Qualidade da atenção pré-natal na rede básica de saúde do Brasil: indicadores e desigualdades sociais. Cad. Saúde Pública. 2017; 33(3): e00195815. doi: https:// doi.org/10.1590/0102-311x00195815.

19. Estrela FM, Silva KKA, Cruz MA, Gomes NP. Gestantes no contexto da pandemia da Covid-19: reflexões e desafios. Physis: Rev Saúde Coletiva. 2020; 30(2): e300215. doi: https://doi.org/10.1590/S0103-73312020300215.

20. Silva RCF, Souza BF, Wernet M, Fabbro MRC, Assalin ACB, Bussadori JCC. Satisfação no parto normal: encontro consigo. Rev Gaúcha Enferm. 2018; 39: e20170218. doi: https://doi.org/10.1590/1983-1447.2018.20170218.

21. Lira IMS, Melo SSS, Gouveia MTO, Feitosa VC, Guimarães TMM. Intervenção educativa para melhorar a frequência ao parto normal. Enf glob. 2020 Abr; 19(58): 226-256. doi: https://doi.org/10.6018/eglobal.382581.

22. Pinto IR, Martins VE, Oliveira JF, Oliveira KF, Paschoini MC, Ruiz MT. Adesão à consulta puerperal: facilitadores e barreiras. Esc. Anna Nery. 2021; 25(2): e20200249. doi: http://dx.doi.org/10.1590/2177-9465-ean-2020-0249.

\section{How to cite this article/Como citar este artigo:}

Ferreira BA, Silva EM. Belarmino AC, Franco RGFM, Sombra ICN, Freitas ASF. PIntegralidade do cuidado de enfermagem do pré-natal ao puerpério. J Health Biol Sci. 2021; 9(1):1-6. 